\title{
From valence bond solid to unconventional superconductivity in the organic charge-transfer solids *
}

\author{
S. Mazumdar ${ }^{\mathrm{a}, 1}$ R.T. Clay ${ }^{\mathrm{b}} \mathrm{H} . \mathrm{Li}^{\mathrm{a}}$ \\ a Department of Physics, University of Arizona Tucson, AZ 85721 \\ ${ }^{\mathrm{b}}$ Department of Physics and Astronomy and HPC ${ }^{2}$ Center for Computational Sciences, Mississippi State University, \\ Mississippi State MS 39762
}

\begin{abstract}
We show that superconductivity is absent within the $\frac{1}{2}$-filled band triangular lattice repulsive Hubbard model that has been proposed for organic charge-transfer solids. We posit that organic superconductivity is rather reached from a Bond-Charge Density Wave that either constitutes the insulating state proximate to superconductivity, or is extremely close in energy to the antiferromagnetic state, and replaces the latter under pressure. The Bond-Charge Density Wave can be described within an effective attractive $U$ extended Hubbard Hamiltonian with repulsive nearest neighbor interaction $V$. A first-order transition from the insulating to the superconducting state occurs within the model with increasing frustration.
\end{abstract}

\section{Introduction}

Superconductivity (SC) in the organic chargetransfer solids (CTS) is proximate to a number of spatial broken symmetry states, including antiferromagnetism (AFM), the spin-Peierls (SP) state and charge-ordering (CO). There are two goals of our presentation. First, we show from exact diagonalization that SC is absent within the $\frac{1}{2}$-filled band triangular lattice repulsive Hubbard model for any parameters. This model Hamiltonian has been proposed to explain the behavior of $\kappa$-ET and related systems in which SC is proximate to AFM. Meanfield calculations find narrow regions of SC between AFM and PM phases 12. We show from exact calculations of pair-pair correlations that $\mathrm{SC}$ is absent within the model for any anisotropy or electronelectron (e-e) interaction. Second, we present evidence that the insulator-superconductor transition

\footnotetext{
* Supported by DOE Grant No. DE-FG02-06ER46315 and NSF Grant No. DMR-0705163.

1 email: sumit@physics.arizona.edu
}

in the CTS is a transition from a Bond-Charge Density Wave (BCDW). We point out that the BCDW is identical to the Valence Bond Solid (VBS) found recently in the $\mathrm{EtMe}_{3}\left[\mathrm{Pd}(\mathrm{dmit})_{2}\right]_{2}$ compound 3$]$. We argue that the $\frac{1}{4}$-filled band $\mathrm{BCDW} / \mathrm{VBS}$ can be modeled by an effective $\frac{1}{2}$-filled band negative- $U$ Hubbard model with nearest neighbor repulsion $V$. With increasing frustration a transition from the VBS to SC occurs within the model. We discuss implications of this work for the CTS.

\section{The repulsive- $U$ Hubbard model}

We consider the Hamiltonian

$$
\begin{aligned}
H & =-t \sum_{\langle i j\rangle, \sigma}\left(c_{i, \sigma}^{\dagger} c_{j, \sigma}+H . c .\right)-t^{\prime} \sum_{[k l], \sigma}\left(c_{k, \sigma}^{\dagger} c_{l, \sigma}\right. \\
& +H . c .)+U \sum_{i} n_{i, \uparrow} n_{i, \downarrow} .
\end{aligned}
$$

In Eq. 1 $c_{i, \sigma}^{\dagger}$ creates an electron with spin $\sigma(\uparrow, \downarrow)$ on site $i, n_{i, \sigma}=c_{i, \sigma}^{\dagger} c_{i, \sigma} . U$ is the on-site e-e interaction. The lattice structure is the conventional square lat- 


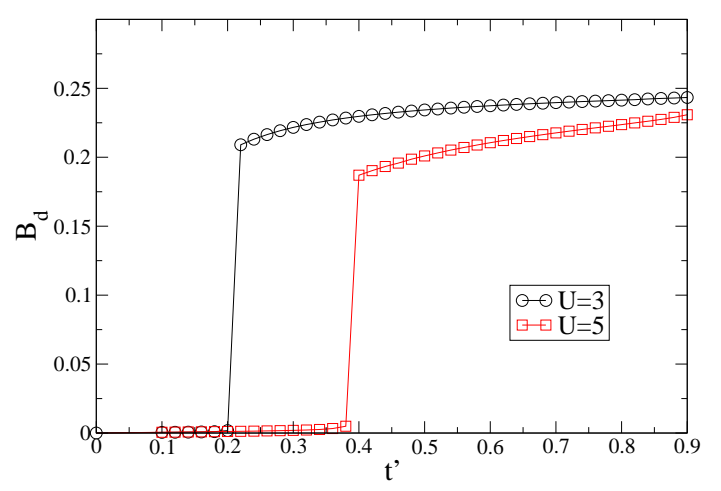

Fig. 1. Diagonal bond order $B_{d}$ for $U=3$ and $U=5$. The jump in $B_{d}$ indicates a transition to a metallic state.

tice (hopping integrals $t$ ) with additional hopping integrals $t^{\prime}$ across the $x+y$ diagonals for a total of three bonds per site. The ratio $t^{\prime} / t$ interpolates from the square lattice $\left(t^{\prime} / t=0\right)$ to the isotropic triangular lattice $\left(t^{\prime} / t=1\right)$. In what follows, we express all quantities in units of $t$.

We report results of exact diagonalizations for $\frac{1}{2}$ filling within a periodic $4 \times 4$ lattice. For $t^{\prime}=0$, the ground state of Eq. 1 has long range AFM for any nonzero $U$. With nonzero $t^{\prime}$, the ground state is a PM until $U>U_{c}\left(t^{\prime}\right)$, when the ground state is an AFM semiconductor. Details of the insulating and magnetic phases can be found elsewhere 415]. Here we focus on the insulator-metal transition and possible superconducting behavior near the boundary.

The insulator-metal transition can be understood from the behavior of the bond-order $B(i, j)$ between sites $i$ and $j$, which measures the probability of charge-transfer and is defined as,

$B(i, j)=\sum_{\sigma}\left\langle c_{i, \sigma}^{\dagger} c_{j, \sigma}+H . c.\right\rangle$

$B(i, j)$ is expected to be large (small) in the metallic (insulating) phase. We define the standard paircreation operator $\Delta_{i}^{\dagger}$,

$\Delta_{i}^{\dagger}=\frac{1}{\sqrt{2}} \sum_{\nu} g(\nu)\left(c_{i, \uparrow}^{\dagger} c_{i+\nu, \downarrow}^{\dagger}-c_{i, \downarrow}^{\dagger} c_{i+\nu, \uparrow}^{\dagger}\right)$

In Eq. 3 the phase factor $g(\nu)$ is (a) +1 for the four nearest-neighbor sites $\nu=i+\hat{x}, i+\hat{y}, i-\hat{x}$ and $i-\hat{y}$ for $s$-wave pairing; (b) $+1,-1,+1,-1$ for the same four sites for $d_{x^{2}-y^{2}}$ pairing; and (c) alternates sign over the four sites $i+\hat{x}+\hat{y}, i+\hat{x}-\hat{y}, i-\hat{x}-\hat{y}$, $i-\hat{x}+\hat{y}$ for $d_{x y}$ pairing. We have calculated pair-pair correlation functions $P(r)=\left\langle\Delta_{i}^{\dagger} \Delta_{i+\vec{r}}\right\rangle$ as a function of distance $r$ for all these symmetries as well as their superpositions. Here we report our results for only

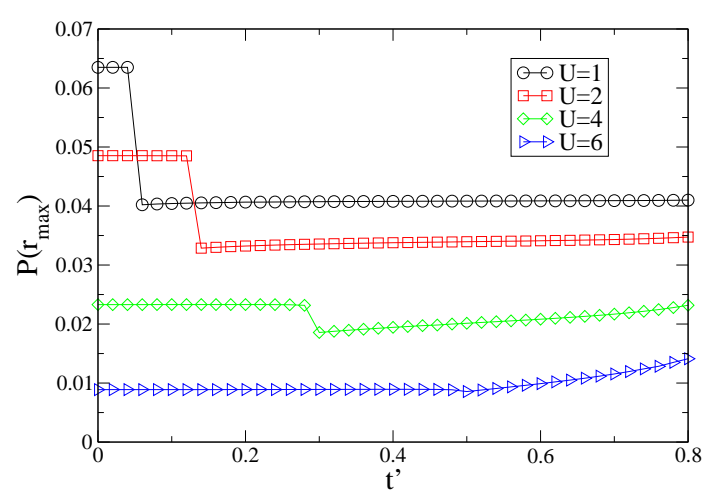

Fig. 2. $P\left(r_{\max }=2 \sqrt{2}\right)$ as a function of $t^{\prime}$ for $U=1,2,4,6$. Long-range pairing is suppressed by $U$ at all $t^{\prime}$.

the $d_{x^{2}-y^{2}}$ pairing, which is supposed to dominate for $U>0$ and $t^{\prime}<1$ [12].

In Fig. 1 we have plotted $B(i, j)=B_{d}$ for $j=$ $i+\hat{x}+\hat{y}$ against $t^{\prime}$ for two different $U . B_{d}$ is a measure of "metallicity". Our results correctly demonstrate the validity of the finite size calculations, with $B_{d} \sim$ 0 for small $t^{\prime}$ for both $U=3$ and 5 , as expected for the insulating regime, and $B_{d}$ showing a sudden jump to a large value at a $U$-dependent $t^{\prime}$, where a transition has occurred to either to the PM phase or to a SC state. We have found similar behavior for other $U, 1 \leq U \leq 10$.

A necessary condition for $\mathrm{SC}$ within the $U>0$ model is that there exists at least a range of $U$ within which $P(r)$ at fixed $r$ is enhanced by $U$. In Fig. 2 we have plotted $P_{d_{x^{2}-y^{2}}}(r=2 \sqrt{2})$ against $t^{\prime}$ for several values of $U$. As seen in this figure, there occurs a monotonic decrease in the pair-pair correlation function with $U$ for any $t^{\prime}$. This is a clear and convincing proof that any tendency to pairing is destroyed by $U$. We have obtained identical results for $s$ and $d_{x y}$ pairing.

\section{VBS and the effective attractive $U$ model}

An alternate theoretical description of superconducting CTS and related systems begins from the number of carriers per molecular site $\rho=\frac{1}{2}$, and emphasizes their $\frac{1}{4}$-filled band nature $[6 / 7 / 8|9| 10$. The appropriate theoretical model in this case is the $\frac{1}{4}$ filled band extended Hubbard model, with nonzero nearest neighbor Coulomb repulsion $V$, as well as the intersite and intrasite electron-phonon (e-p) interactions. We have solved this Hamiltonian for the quasi-1D (TMTTF $)_{2} \mathrm{X}[6$, the zigzag ladder system DTTTF-Cu(mnt $)_{2}$ [7, the quasi-2D (TMTSF $)_{2} \mathrm{X}$ [8] and $\theta-\mathrm{ET}_{2} \mathrm{X}$ [9]. Two different $\mathrm{CO}$ patterns are 
(a)

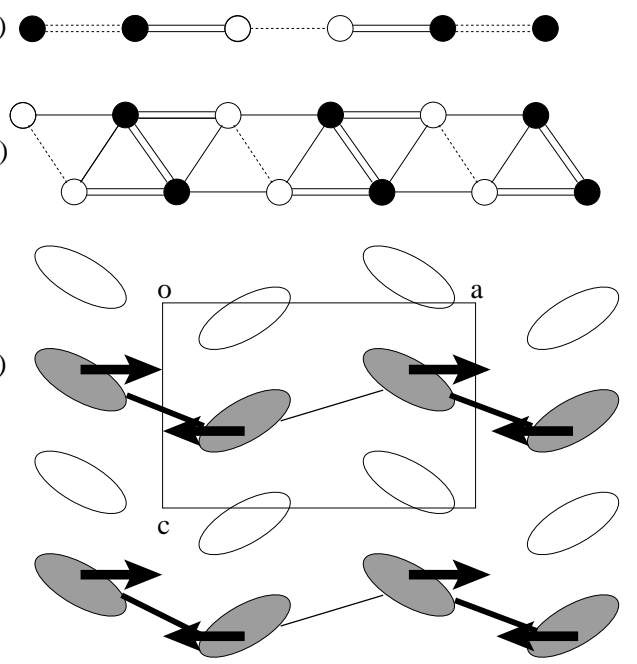

Fig. 3. (a) The ...1100... 1D BCDW state; (b) The zigzag ladder BCDW state; (c) horizontal stripe CO in $\theta-(E T)_{2} X$ with SP distortion at low temperature (see reference [1]). Filled (open) symbols represent charge-rich (charge-poor) sites in all cases.

possible in these compounds: the Wigner crystal, with charge-rich sites having only charge-poor sites as neighbors; and the BCDW/VBS, in which the density wave consists of periodic arrangements of pairs of charge-rich sites that form local singlets or bipolarons. The BCDW results from a co-operative interaction between the e-p interactions and the AFM coupling, and dominates for realistic $U \sim 6-8$ and $V \sim 1-36778910$.

In Fig. 3 we have shown the BCDW for the 1D chain, the zigzag ladder, and the $\theta$-ET triangular lattice with the dominant horizontal stripe CO. In all these cases the $\rho=\frac{1}{2}$ BCDW can be modeled as an effective $\rho=1$ lattice, with each site of the effective lattice composed of pairs of occupied or unoccupied molecular sites. This is most obvious in the $1 \mathrm{D}$ case in Fig. 3 (a), where the $\rho=\frac{1}{2} \cdots 1100 \cdots$ BCDW (here ' 1 ' and ' 0 ' denote the charge-rich and charge-poor sites with actual site charges $0.5+\epsilon$ and $0.5-\epsilon$, respectively.) can clearly be thought of as the $\rho=1$ density wave $\cdots 2020 \cdots$ (with ' 2 ' and ' 0 ' having charges $1.0+\epsilon$ and $1.0-\epsilon$, respectively.)

\section{Negative- $U$ extended Hubbard model}

We model such a system by an effective negative$U$ extended Hubbard Hamiltonian [10],

$$
H=-t \sum_{\langle i j\rangle, \sigma}\left(c_{i, \sigma}^{\dagger} c_{j, \sigma}+H . c .\right)-t^{\prime} \sum_{[k l], \sigma}\left(c_{k, \sigma}^{\dagger} c_{l, \sigma}+\right.
$$

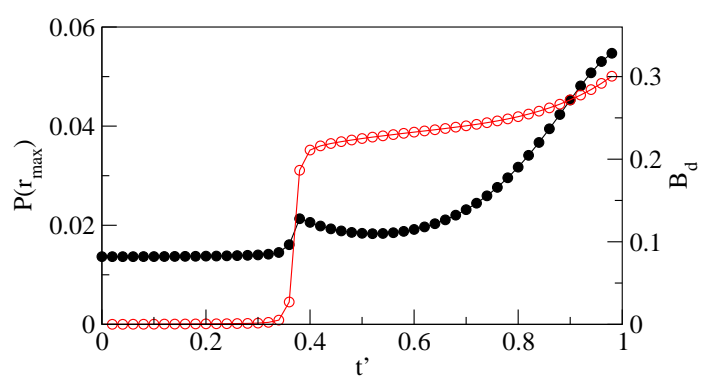

Fig. 4. $B_{d}$ (open symbols) and $P\left(r_{\max }\right)$ (filled symbols) as a function of $t^{\prime}$ in the negative- $U$ model for $U=-1$, $V=V^{\prime}=1$. Transition to SC occurs at $t^{\prime}=0.38$.

$$
\text { H.c. })-|U| \sum_{i} n_{i, \uparrow} n_{i, \downarrow}+V \sum_{\langle i j\rangle} n_{i} n_{j}+V^{\prime} \sum_{[k l]} n_{k} n_{l}
$$

All terms have their usual meanings, with $n_{i}=$ $\sum_{\sigma} n_{i, \sigma} ;\langle\ldots\rangle$ implies n.n. along the $\hat{x}$ - and $\hat{y}$-axes, with hopping integral and Coulomb repulsion $t$ and $V$, respectively. Similarly, [...] implies neighbors along the $(\hat{x}+\hat{y})$-diagonal, with $t^{\prime}$ and $V^{\prime}$ as the hopping and Coulomb integrals. We emphasize that the effective attractive $U$ here originates from the co-operative interaction between the AFM coupling and e-p interactions in the original repulsive- $U$ $\frac{1}{4}$-filled band Hamiltonian.

For small $t^{\prime}$ and $V^{\prime}$, the ground state of Eq. 4 is the checkerboard $\mathrm{CO}$, with double occupancies as neighbors of vacancies and vice versa. We have done exact diagonalizations, again on the periodic $4 \times 4$ lattice, that finds a sharp CO-to-SC transition within the model with increasing frustration. The pair-correlation function here is defined as,

$P(r)=\frac{1}{N} \sum_{j}\left\langle c_{j, \uparrow}^{\dagger} c_{j, \downarrow}^{\dagger} c_{j+\vec{r}, \downarrow} c_{j+\vec{r}, \uparrow}\right\rangle$

For each combination of $U, V$ and $V^{\prime}$ we have found that $P(r)$ exhibits LRO (to the extent that this can be measured on a finite lattice) for $t^{\prime}$ greater than a critical value. This is shown in Fig. 4 for the specific case of $U=-1, V=V^{\prime}=1$, where we have plotted both $B_{d}$ and $P\left(r_{\max }=2 \sqrt{2}\right)$ versus $t^{\prime}$. The behavior of $B_{d}$ is similar to that in Fig. 1, with a semiconducting state for $t^{\prime}<0.38$. From calculations of charge-charge correlations and the static structure factor (not shown) we have confirmed that the semiconducting state is the checkerboard CO. The jump in $B_{d}$ at $t^{\prime}=0.38$ is due to the transition to a superconducting state, as evidenced by the simultaneous jump in $P\left(r_{\max }\right)$. Calculations over broad ranges of $U$ and $V^{\prime}$ indicate that the transition is first-order, 
when the initial CO is strong, and second-order otherwise.

\section{Significance for the CTS}

We believe that the negative- $U$ Hubbard Hamiltonian with repulsive $V$ gives the correct insight to understanding the unconventional SC in the organics, in spite of its limitations. To begin with, we believe that the same fundamental mechanism applies to (TMTSF $)_{2} \mathrm{X}, \mathrm{ET}_{2} \mathrm{X}$ and the anionic $\left[\mathrm{Pd}(\text { dimit })_{2}\right]_{2}$ salts. In all these cases the lattice structure is anisotropic triangular. We believe that the role of pressure is to increase the interchain coupling and take the systems towards the more isotropic limit. We present a brief discussion of implications of our work below.

1. Although the insulator-to-SC transition in the organics have been often thought to be an AFMto-SC transition, as shown in section II, there is no $\mathrm{SC}$ within the triangular lattice $\frac{1}{2}$-filled band Hubbard model. It is also significant that AFM is missing in the insulating states of the more isotropic $\kappa$ $\mathrm{ET}_{2} \mathrm{Cu}_{2}(\mathrm{CN})_{3}\left[12\right.$ and $\mathrm{EtMe}_{3}\left[\mathrm{Pd}(\mathrm{dmit})_{2}\right]_{2}$ [3]. As we have already pointed out, the experimentally determined VBS in $\mathrm{EtMe}_{3}\left[\mathrm{Pd}(\text { dmit })_{2}\right]_{2}$ is charge disproportionated and has the same charge-densities and intermolecular distances as in our BCDW [3]. We conclude that even when the insulating state is AFM, pressure-induced frustration leads to a transition to the BCDW, which in turn becomes superconducting.

2. Partial justification of the above proposed scenario comes from our recent work on the temperature-dependence of the spatial broken symmetries in (TMTTF $)_{2} \mathrm{X}$ [6]. As shown in this work, here the high temperature $\mathrm{CO}$ state is a Wigner crystal, but as the temperature is lowered and the system enters the SP state, the BCDW dominates. Similar pressure-induced AFM-to-SP transition here is also accompanied by a transition from the Wigner crystal CO pattern to the BCDW 13. The implication of these experiments is that the BCDW is very close energetically to the other broken symmetry states even when the latter dominate.

3. Very large upper critical field is a characteristic of the superconducting CTS. This and the upward curvature of $\mathrm{T}_{c}$ versus magnetic field is a characteristic of superconductors with local pairing [14.

4. Our theoretical model is a "superposition" of the t-J model (the intersite pairing being driven by AFM correlations) and the so-called bipolaron model of SC 14]. A critical difference from the standard bipolaron model is the crucial role of the number of carriers per site $\rho=\frac{1}{2}$, and the resulting co-operation, as opposed to competition, between e-e and e-p interactions. Both $\rho=\frac{1}{2}$ and frustration are essential ingredients of the insulator-tosuperconductor transition.

5. The bipolaron model has often been criticized because of the supposedly heavy masses of the bipolarons. This, however, is strictly true only for the on-site bipolarons and not for intersite bipolarons. The latter are particularly mobile on triangular lattices 15 .

6. The superconducting symmetry within the negative- $U$ extended Hubbard Hamiltonian can only be s-wave. This, however, need not to be true within the actual $\frac{1}{4}$-filled band repulsive $U$ extended Hubbard Hamiltonian that provides a more complete description of the CTS 678910]. The question of gap symmetry can therefore be resolved only after the true Hamiltonian is solved. Work is under progress in this direction.

\section{References}

[1] B. J. Powell, R. H. McKenzie, Phys. Rev. Lett. 94 (2005) 047004.

[2] B. Kyung, A. M. S. Tremblay, Phys. Rev. Lett. 97 (2006) 046402.

[3] R. Kato, A. Tajima, A. Nakao, M. Tamura, J. Am. Chem. Soc. 128 (2006) 10016.

[4] T. Mizusaki, M. Imada, Phys. Rev. B 74 (2006) 014421.

[5] R. T. Clay, H. Li, S. Mazumdar, preprint arXiv:0805.0590 (2008).

[6] R. T. Clay, R. P. Hardikar, S. Mazumdar, Phys. Rev. B 76 (2007) 205118.

[7] R. T. Clay, S. Mazumdar, Phys. Rev. Lett. 94 (2005) 207206.

[8] S. Mazumdar, S. Ramasesha, R. T. Clay, D. K. Campbell, Phys. Rev. Lett. 82 (1999) 1522.

[9] R. T. Clay, S. Mazumdar, D. K. Campbell, J. Phys. Soc. Jpn. 71 (2002) 1816.

[10] S. Mazumdar, R. T. Clay, Phys. Rev. B 77 (2008) 180515(R).

[11] M. Watanabe, Y. Noda, Y. Nogami, H. Mori, J. Phys. Soc. Jpn. 76 (2007) 124602.

[12] Y. Shimizu, K. Miyagawa, K. Kanoda, M. Maesato, G. Saito, Phys. Rev. Lett. 91 (2003) 107001.

[13] W. Yu, et al., Phys. Rev. B 70 (2004) 121101.

[14] R. Micnas, J. Ranninger, S. Robaszkiewicz, Rev. Mod. Phys. 62 (1990) 113.

[15] J. P. Hague, P. E. Kornilovitch, J. H. Samson, A. S. Alexandrov, Phys. Rev. Lett. 98 (2007) 037002. 